\title{
Philosophiques
}

\section{Peter Van Inwagen. Thinking about Free Will ?, Cambridge, Cambridge University Press, 2017, 232 pages}

\section{Simon-Pierre Chevarie-Cossette}

Volume 44, numéro 2, automne 2017

URI : https://id.erudit.org/iderudit/1042347ar

DOI : https://doi.org/10.7202/1042347ar

Aller au sommaire du numéro

Éditeur(s)

Société de philosophie du Québec

ISSN

0316-2923 (imprimé)

1492-1391 (numérique)

Découvrir la revue

Citer ce compte rendu

Chevarie-Cossette, S.-P. (2017). Compte rendu de [Peter Van Inwagen. Thinking about Free Will ?, Cambridge, Cambridge University Press, 2017, 232 pages].

Philosophiques, 44(2), 410-414. https://doi.org/10.7202/1042347ar

Ce document est protégé par la loi sur le droit d'auteur. L'utilisation des services d’Érudit (y compris la reproduction) est assujettie à sa politique d'utilisation que vous pouvez consulter en ligne.

https://apropos.erudit.org/fr/usagers/politique-dutilisation/ 
Peter Van Inwagen. Thinking about Free Will?, Cambridge, Cambridge University Press, 2017, 232 pages.

Dans Thinking about Free Will, Peter van Inwagen présente une synthèse de sa contribution philosophique au débat sur le libre arbitre. Le livre regroupe quatorze chapitres, dont onze déjà publiés tels quels, classés en ordre chronologique de publication. Il y a lieu de regretter cette frugalité en nouveau matériel; d'abord bien sûr parce que van Inwagen écrit avec clarté et pénétration, mais aussi parce que les chapitres réimprimés ne sont ni retravaillés ni commentés individuellement. Van Inwagen nous offre plutôt une étrange (mais amusante) introduction où il relate à la troisième personne l'évolution de ses idées sur le libre arbitre. Quoi qu'il en soit, ceux qui craindront de découvrir, dans les premiers chapitres, des arguments périmés ou abandonnés seront rassurés: depuis son célèbre livre de 1983 , van Inwagen n'a à peu près pas modifié son credo sur le libre arbitre. Les rares exceptions à cette continuité sont énumérées à notre plus grand profit dans le douzième chapitre $^{2}$.

Ce credo se décline en quatre thèses principales, qui permettent de restructurer l'ouvrage thématiquement. La première thèse est l'incompatibilité du déterminisme et du libre arbitre (l'incompatibilisme), une thèse en faveur de laquelle il a donné l'un des meilleurs arguments, l'argument de la conséquence (voir chap. I, 7, 11; chap. II, 9, pour des réponses aux compatibilistes). Thinking about Free Will présente à peu près tout ce que van Inwagen a offert sur cette question dans sa carrière philosophique.

La seconde thèse est que le libre arbitre semble aussi incompatible avec l'indéterminisme. Van Inwagen récupère le Mind Argument ${ }^{3}$ : si l'indéterminisme est nécessaire au libre arbitre, c'est parce que les actions libres sont indéterminées; or, si une action est indéterminée, elle est le fruit du hasard et n'est donc pas libre; donc, l'indéterminisme n'est pas nécessaire au libre arbitre. Bien qu'il n'en soit pas l'auteur, van Inwagen soutient le Mind Argument en proposant une réponse à ses opposants (voir chap. VII). Ceux-ci refusent l'opposition entre hasard et détermination. Ils avancent parfois que les actions humaines sont indéterminées au sens où elles sont causées par des substances indéterminées (les humains). Van Inwagen persiste et signe: si nous sommes des substances indéterminées, il semble être hors de notre contrôle que nous engendrions telle ou telle chaîne causale.

La conséquence logique des deux premières thèses est l'inexistence du libre arbitre, mais van Inwagen s'y refuse. Il favorise une troisième thèse: la question du libre arbitre est un mystère (voir chap. VII). Van Inwagen nous

1. Peter van Inwagen, An Essay on Free Will, Oxford, Oxford University Press, 1983.

2. La version française de ce chapitre sera la préface de Peter van Inwagen, Un essai sur le libre arbitre, Paris, Vrin, à paraître.

3. Le Mind Argument porte le nom de la revue où il a été défendu. 
assure qu'il ne s'agit pas d'un mystère théologique, mais plutôt d'un puzzle philosophique comme l'a longtemps été le paradoxe de Zénon. Van Inwagen suspecte que c'est le Mind Argument qui est erroné, mais il s'avoue vaincu. Il a même l'audace de suggérer que cette entreprise dépasse l'entendement humain (p. 15). Si tel est le cas, le mystère théologique semble refaire surface.

La quatrième thèse, mi-métaphysique, mi-méthodologique, est que le libre arbitre doit être compris comme la "capacité (ability) de faire autrement » (voir chap. I, 8, 10,13,14). Il faut s'y attarder puisque les nouveaux chapitres y sont directement liés. La capacité de faire autrement se différencie d'abord de la situation où nous sommes placés devant une alternative ( "to have alternative possibilities»). Van Inwagen croit que c'est une erreur qui résulte bêtement de la polysémie du mot «could» dans "could have done otherwise (voir chap. IV). Nombreux sont ceux qui auraient alors conclu à tort qu'il y a deux concepts de libre arbitre, celui des compatibilistes (qui signifie être la source de ses actions) et celui des incompatibilistes (qui signifie être devant une alternative) ${ }^{4}$. Une fois commise, cette erreur en entraîne une autre: rejeter le libre arbitre incompatibiliste et admettre que le compatibilisme est vrai. En effet, comme l'a proposé Harry Frankfurt ${ }^{5}$, un agent pourrait ne plus être placé devant une alternative sans pour autant que sa responsabilité morale soit sapée si ce qui l'empêche d'être devant une alternative ne cause pas son action. Comme la plupart des philosophes qui travaillent sur le libre arbitre postulent que celui-ci est nécessaire à la responsabilité, le libre arbitre incompatibiliste est alors jugé problématique.

Une fois que l'on fait marche arrière et que l'on conçoit le libre arbitre comme une capacité, les remarques de Frankfurt peuvent sembler hors sujet. Van Inwagen peut maintenir que la capacité d'agir autrement est nécessaire à la responsabilité, mais cela ne signifie pas que pour être responsable de $x$, il faut pouvoir éviter $x$ au moment de l'action. L'important est simplement que nous ayons la capacité à un moment de nos vies d'éviter de faire advenir les états de fait dont nous sommes responsables. En réalité, la majorité de nos actions ne sont pas libres (voir chap. V): par exemple, je ne suis pas libre de m'acheter une voiture si je ne vois aucune raison de le faire. Mes seules décisions réellement libres sont celles où la balance ne penche pas sérieusement d'un côté. Néanmoins, je puis être responsable des conséquences de ne pas avoir acheté une voiture puisqu'il y a eu un moment où j'avais la capacité de changer certaines de mes raisons. C'est à mon avis donner beaucoup trop de poids à notre passé - bien qu'il puisse en partie être pertinent lorsqu'il s'agit d'évaluer notre responsabilité. (Soit, nous sommes respon-

4. Voir, par exemple, Daniel Dennett, Elbow Room: The Varieties of Free Will Worth Wanting, Cambridge, MIT Press, 1984.

5. Harry Frankfurt, «Alternate Possibilities and Moral Responsibility », The Journal of Philosophy, vol. 66, n² 22, 1969. 
sables des conséquences d'actions violentes commises sous l'influence de l'alcool parce que nous aurions pu nous abstenir de boire. Cela n'implique pas que nous soyons responsables des conséquences de nos valeurs parce que nous aurions pu en adopter d'autres pendant l'adolescence.)

Aujourd'hui, les débats sur le libre arbitre supposent presque toujours qu'il y a plusieurs notions concurrentes de libre arbitre et que le débat entre les partisans de chaque conception est un réel enjeu philosophique ${ }^{6}$. Van Inwagen croit au contraire que ces débats ne sont que d'absurdes joutes verbales (p. 194), puisque le terme free will est un terme technique. C'est un cas «d'essentialisme verbal ", c'est-à-dire que les parties du débat communiquent quelque chose qu'elles ne pourraient exprimer si elles n'avaient pas accès au mot «libre arbitre»(p. 202). En conséquence, van Inwagen nous exhorte à repenser le problème du libre arbitre sans les expressions free will, freedom, et could have (voir chap. X et XIII). Van Inwagen marque un point: si un débat sur la nature d'un concept comme l'égalité ou le courage peut être réellement philosophique, les débats sur la nature de concepts techniques semblent parfois inconséquents. De fait, rien d'emblée ne semble empêcher l'admission des deux concepts; il ne faut que s'entendre sur qui "aura droit" au mot en question. Bien sûr, on peut penser qu'il y a un sens stratégique à déterminer, par exemple, si la race noire existe et donc qu'elle est une catégorie socialement imposée, ou si au contraire elle n'existe pas puisque les races sont biologiques. Cela n'en fait pas d'emblée un débat philosophique.

Malgré tout, van Inwagen fait fausse route. Même si certains débats sur la nature de concepts techniques ne sont pas philosophiques, cela est loin d'être évident en ce qui concerne la question du libre arbitre. D'abord, on peut déjà remettre en doute la frontière entre termes techniques et termes courants - après tout, "liberté " n'est pas qu'un terme technique, et la question du libre arbitre accompagne la philosophie depuis si longtemps qu'elle a d'une certaine façon pénétré le grand public. Ensuite, et surtout, un débat sur la nature du libre arbitre est important puisque le libre arbitre a un ancrage fonctionnel qui permet aux intervenants dans le débat d'être certains de parler de la même chose. Cet ancrage fonctionnel, c'est la responsabilité morale; le libre arbitre est la capacité de contrôle nécessaire à la responsabilité morale ${ }^{7}$.

Van Inwagen est tout à fait conscient de cet ancrage, aussi son diagnostic d'essentialisme verbal est-il non seulement erroné, mais surprenant.

6. Voir, par exemple, Susan Wolf, Freedom within Reasons, Oxford, Oxford University Press, 2003; et Derk Pereboom, Free Will, Agency, and Meaning in Life, Oxford, Oxford University Press, 2014.

7. Voir, par exemple, Gregg D. Caruso et Stephen G. Morris, «Compatibilism and Retributivist Desert Moral Responsibility: On What is of Central Philosophical Importance ", vol. 82, n 4, Erkenntnis, 2016. 
Van Inwagen peut délaisser «libre arbitre» au profit de "capacité à faire autrement "; de la même manière, les parties dans le débat peuvent très bien se délester des mots "libre» et "libre arbitre », et utiliser la périphrase "capacité de contrôle nécessaire à la responsabilité morale». La joute verbale se situe donc, non pas entre les philosophes que van Inwagen critique, mais entre eux et van Inwagen lui-même. Quel est le débat qui nous intéresse et comment le formuler?

La deuxième définition fonctionnelle est plus œcuménique. Elle rassemble en effet non seulement ceux qui croient que la capacité de faire autrement est nécessaire à la responsabilité morale, mais aussi ceux qui croient le contraire $^{8}$. En effet, ceux qui croient que la responsabilité n'a rien à voir avec la capacité de faire autrement voient peu d'intérêt à se poser la question de la compatibilité entre le déterminisme et cette capacité. La définition fonctionnelle permet aussi à ceux qui croient que le déterminisme est compatible avec la capacité de faire autrement, mais pas avec la responsabilité morale, de faire partie du clan des incompatibilistes, ce qui semble naturel. Bref, refuser la proposition de van Inwagen permet de convier plus de participants au débat. Van Inwagen croit que sa définition a l'avantage de suivre la tradition et d'être plus claire (p. 188). Elle est certainement plus claire au sens où elle est plus restreinte et donc moins sujette à être débattue. Par contre, l'avantage de la tradition se limite à la période de 1960 à 1980, que van Inwagen appelle, non sans aplomb, «l'ère classique ». De fait, le concept de libre arbitre a évolué depuis Saint-Augustin! Ce débat est somme toute terminologique, puisqu'autant van Inwagen que ses opposants peuvent exprimer leur position dans le langage préféré de l'autre partie, quoique l'exercice de traduction puisse être fécond. Dans tous les cas, les accusations de van Inwagen tombent.

Le quatorzième et dernier chapitre, toujours lié à la capacité de faire autrement, est un véritable vent de fraîcheur. Van Inwagen nous entraîne directement sur le terrain qu'il connaît admirablement: celui de la nature des habiletés. Après une brève histoire des débats sur le libre arbitre pendant "l'ère classique ", van Inwagen se propose de nous expliquer la notion précise d'habileté impliquée dans la notion «classique» de libre arbitre. Il y a en effet un sens dans lequel une pianiste a toujours la capacité de jouer du piano lorsqu'elle prend l'avion; mais ce n'est manifestement pas un sens qui est pertinent pour la responsabilité morale. Imaginons qu'au décollage des enfants pleurent. Il devient rapidement évident que la seule façon de les calmer serait de leur jouer de la musique. Le fait que notre pianiste a la capacité de jouer du piano ne la rend pas responsable du fait que les passagers doivent endurer les pleurs!

8. Voir, par exemple, John M. Fischer, "Semicompatibilism and Its Rivals", The Journal of Ethics, vol. 2, nº 16, 2012. 
Van Inwagen croit que le sens pertinent de la capacité à faire autrement peut être dégagé en réfléchissant sur les promesses défectueuses. Si un médecin ne croit pas pouvoir sauver un patient et promet à l'épouse de ce dernier qu'il le fera, c'est une promesse défectueuse (du moins en tant que promesse, et donc qu'elle soit morale ou non). Pour faire une telle promesse, un médecin doit avoir la croyance qu'il a la capacité de sauver le patient. Le sens minimal de cette capacité qui est nécessaire pour que la promesse du médecin ne soit pas défectueuse serait précisément le sens pertinent pour la responsabilité morale. Cela discrédite l'analyse conditionnelle classique (à la Hume): on n'imaginerait pas une personne terrorisée par les chiens promettre à son voisin de s'occuper de son bull-dog parce qu'elle en a la capacité au sens humien (c'est-à-dire au sens où, si elle en avait la volonté, elle le ferait). L'on aurait pu espérer que van Inwagen utilise ce cadre pour tester les nouvelles analyses conditionnelles inspirées de l'approche humienne'.

La contribution de Thinking about Free Will, au-delà du fait qu'il rassemble plusieurs excellents articles publiés dans un même ouvrage, est restreinte, mais bienvenue. Si nous en remercions son auteur, c'est cependant avec une certaine réserve. Van Inwagen est prompt à faire litière de ses adversaires, affirmant à l'occasion qu'ils ne méritent pas de réplique (p. ex. p. 11). Il avance en outre dans un des chapitres inédits que nul n'a répondu à ses arguments concernant la délibération (p. 184), alors qu'on dénombre au moins onze réponses publiées dans des revues sérieuses entre 1985 et 2016. Van Inwagen affirme aussi qu'il n'y a qu'une réponse sérieuse à son argument de la conséquence, celle de David Lewis ${ }^{10}$, alors qu'il reconnaît lui-même devoir reformuler certains de ces arguments à la suite de réfutations publiées par d'autres. Enfin, il maintient que depuis 1983,

la littérature philosophique sur le libre arbitre [...] a dégénéré en une scolastique stérile (dans le sens péjoratif du terme) [...]. C'est-à-dire qu'il n'y a eu aucun argument ou idée à la fois nouveau et important [ "of any real consequence» ]. Ceux qui ont pris part à la discussion concernant le problème du libre arbitre depuis 1983 connaissent tous les arguments pertinents et les concepts qui sont liés à chaque aspect du problème, et ils débattent de ces arguments et concepts sans ajouter quoi que ce soit de nouveau et d'important à leur propos (p. 187, traduction libre).

Il est juste que l'essentiel des débats contemporains sur la question concerne plutôt la responsabilité morale que la capacité à faire autrement. En revanche, ces acerbes poussées de nostalgie auraient pu être avantageusement écartées. Le lecteur est prévenu.

SIMON-PIERRE CHEVARIE-COSSETTE

University College, Oxford

9. Voir, par exemple, Khadri Vivhelin, «Free Will Demystified: A Dispositional Account ", Philosophical Topics, vol. 32, $\mathrm{n}^{\text {os }} 1$ et 2, 2004.

10. David Lewis, «Are We Free to Break the Laws?", Theoria, vol. 47, n 3, 1981. 